\title{
A short review of some double-parton scattering processes*
}

\author{
Antoni SzCZureK \\ Institute of Nuclear Physics (PAN), Kraków, Poland, \\ Rzeszów University, Rzeszów, Poland
}

\begin{abstract}
A few examples of quickly developing field of double parton scattering are discussed. We present arguments that the production of two pairs of charm quark-antiquark is the golden reaction to study the double parton scattering effects. We discuss briefly also mechanism of single parton scattering and show that it gives much smaller contribution to the $c \bar{c} c \bar{c}$ final state. In this context we discuss also the contribution of perturbative parton-splitting mechanism which should also be added to the conventional DPS mechanism. The presence of the letter leads to collision energy and other kinematical variables dependence of so-called $\sigma_{e f f}$. We briefly discuss production of four jets. We concentrate on estimation of the contribution of DPS for jets widely separated in rapidity. Finally we briefly mention about DPS effects in production of $W^{+} W^{-}$.
\end{abstract}

PACS numbers: 11.80.La,13.87.Ce,14.65.Dw,14.70.Fm

\section{Introduction}

The double-parton scattering was recognized already in seventies and eighties 1, 2, 3, 4, 5, 6, 7, 8, 9. Several estimates of the cross section for different processes have been presented in recent years [10, 11, 12, 13, 14, 15, [16, 17, 18]. The theory of the double-parton scattering is quickly developing $19,20,21,22,23,24,25,26,27,28,29,30$.

It was recognized recently that the production of $c \bar{c} c \bar{c}$ is a very good place to study DPS effects [31]. Here, the quark mass is small enough to assure that the cross section for DPS is large, but large enough that each of the scatterings can be treated within pQCD. In the meantime the LHCb collaboration presented interesting data for simultaneous production of two charmed mesons [32. In Ref. 33] we discussed that the large cross section in 32 is a footprint of double parton scattering.

\footnotetext{
* Presented at Excited QCD 2015, Tatranska Lomnica, Slovakia, 8-14 March 2015
} 


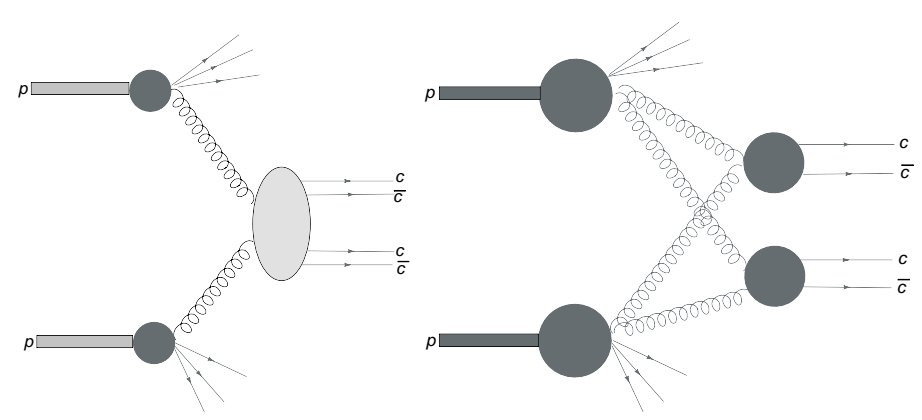

Fig. 1. SPS and DPS production mechanisms of $c \bar{c} c \bar{c}$.

25 years ago Mueller and Navelet predicted strong decorrelation in relative azimuthal angle [34] of jets with large rapidity separation due to exchange of the BFKL ladder between quarks. Since then both leadinglogarithmic [34, 35, 36, 37, 38, 39] and higher-order BFKL effects [40, 41, 42, 43, 44, 45, 46, 47, 48, 49] were calculated and discussed. The effect of the NLL correction is large and leads to significant lowering of the cross section. The LHC opens a new possibility to study the decorrelation in azimuthal angle. First experimental data measured at $\sqrt{s}=7 \mathrm{TeV}$ are expected soon [50]. We discussed recently the contribution of DPS to the jets widely separated in rapidity [51].

The double parton scattering mechanism of $W^{+} W^{-}$production was discussed e.g. in Refs. [11, 52, 53, 54]. The $W^{+} W^{-}$final states constitutes a background to Higgs production. It was discussed recently that doubleparton scattering could explain a large part of the observed signal [55]. Here we discuss briefly the double parton scattering mechanism of $W^{+} W^{-}$ production.

\section{Sketch of the formalism}

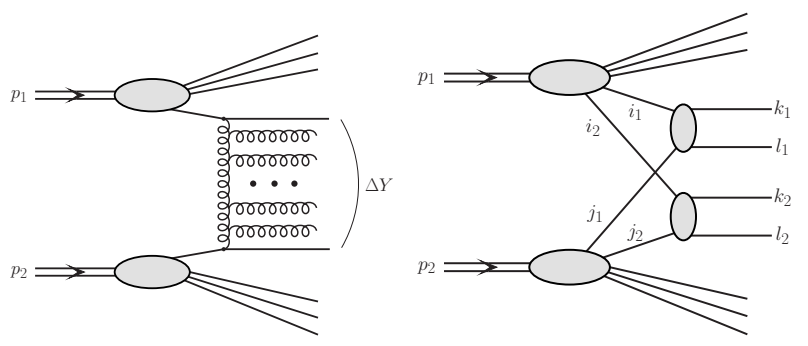

Fig. 2. A diagramatic representation of the Mueller-Navelet jet production (left diagram) and of the double paron scattering mechanism (right diagram). 
Let us consider for example production of $c \bar{c} c \bar{c}$ final state within the DPS framework. In a simple probabilistic picture the cross section for doubleparton scattering can be written as (see [33]):

$$
\sigma^{D P S}(p p \rightarrow c \bar{c} c \bar{c} X)=\frac{1}{2 \sigma_{e f f}} \sigma^{S P S}\left(p p \rightarrow c \bar{c} X_{1}\right) \cdot \sigma^{S P S}\left(p p \rightarrow c \bar{c} X_{2}\right) .
$$

The simple formula (1) can be generalized to address differential distributions. A more general formula for the cross section can be written formally in terms of double-parton distributions, e.g. $F_{g g}, F_{q q}$, etc. (see e.g. [66]).

In the $k_{t}$-factorization approach the differential cross section for DPS production of $c \bar{c} c \bar{c}$ system can be written as:

$$
\begin{aligned}
& \frac{d \sigma^{D P S}(p p \rightarrow c \bar{c} c \bar{c} X)}{d y_{1} d y_{2} d^{2} p_{1, t} d^{2} p_{2, t} d y_{3} d y_{4} d^{2} p_{3, t} d^{2} p_{4, t}}= \\
& \frac{1}{2 \sigma_{e f f}} \cdot \frac{d \sigma^{S P S}\left(p p \rightarrow c \bar{c} X_{1}\right)}{d y_{1} d y_{2} d^{2} p_{1, t} d^{2} p_{2, t}} \cdot \frac{d \sigma^{S P S}\left(p p \rightarrow c \bar{c} X_{2}\right)}{d y_{3} d y_{4} d^{2} p_{3, t} d^{2} p_{4, t}} .
\end{aligned}
$$

In Fig. 3 we illustrate a conventional and perturbative splitting DPS mechanisms for $c \bar{c} c \bar{c}$ production. The $2 \mathrm{v} 1$ single parton splitting mechanism (the second and third diagrams in the figure) were considered first in [57].
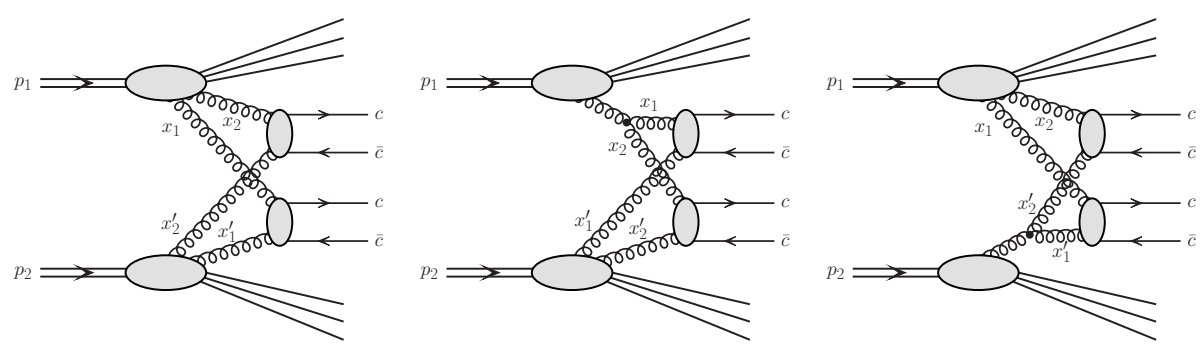

Fig. 3. The diagrams for DPS production of $c \bar{c} c \bar{c}$.

Some more details of the formalism are given e.g. in [66].

\section{Examples of results}

In Fig. 4 we compare cross sections for the single and double-parton scattering as a function of proton-proton center-of-mass energy. At low energies the single-parton scattering dominates. For reference we show the protonproton total cross section as a function of collision energy as parametrized in Ref. 63]. At low energy the $c \bar{c}$ or $c \bar{c} c \bar{c}$ cross sections are much smaller than the total cross section. At higher energies both the contributions approach 

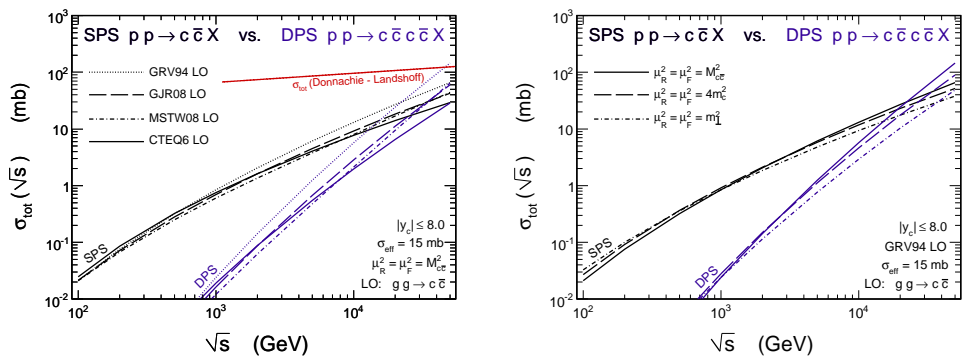

Fig. 4. Total LO cross section for $c \bar{c}$ and double-parton scattering production of $c \bar{c} c \bar{c}$ as a function of center-of-mass energy (left panel) and uncertainties due to the choice of (factorization, renormalization) scales (right panel). We show in addition a parametrization of the total cross section in the left panel.

the total cross section. At LHC energies the cross section for both terms become comparable. This is a completely new situation.

So far we have concentrated on DPS production of $c \bar{c} c \bar{c}$ and completely ignored SPS production of $c \bar{c} c \bar{c}$. In Refs. 61, 62] we calculated the SPS contribution in high-energy approximation [61] and including all diagrams in the collinear-factorization approach 62. In Fig. 5 we show the cross section from Ref. 62. The corresponding cross section at the LHC energies is more than two orders of magnitude smaller than that for $c \bar{c}$ production and much smaller than the DPS contribution.

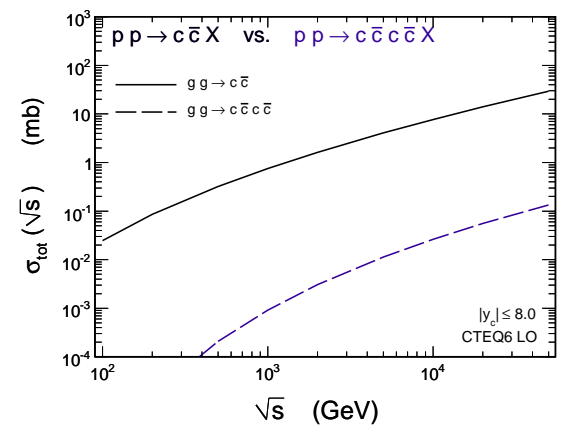

Fig. 5. Cross section for SPS production of $c \bar{c} c \bar{c}$ compared to this for standard $c \bar{c}$ production as a function of collision energy.

In real experiments one measures rather $D$ mesons than charm quarks/antiquarks. In Fig. 6 we show resulting distributions in rapidity distance between two $D^{0}$ mesons (left panel) and corresponding distribution in relative azimuthal angle (right panel). The DPS contribution (dashed line) dominates over the 


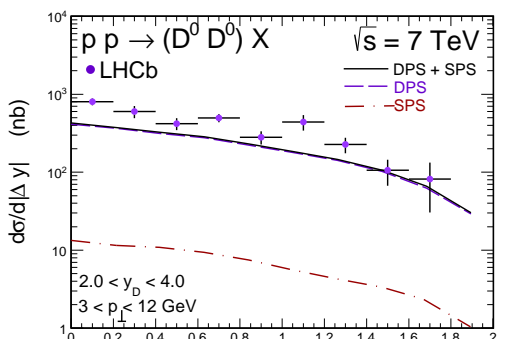

$|\Delta y|$

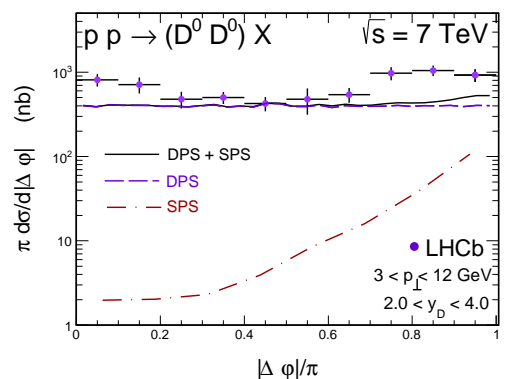

$|\Delta \varphi| / \pi$

Fig. 6. Rapidity distance between two $D^{0}$ mesons (left panel) and corresponding azimuthal correlations (right panel).

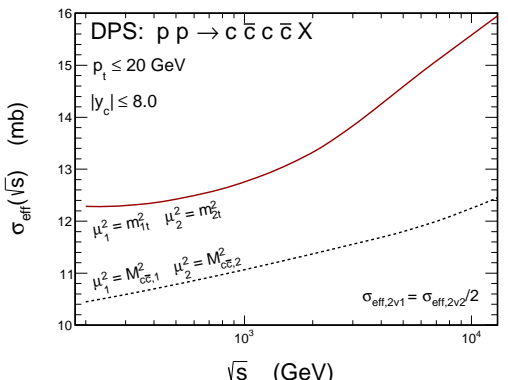

Fig. 7. Energy and factorization scale dependence of $\sigma_{e f f}$ for $c \bar{c} c \bar{c}$ production as a consequence of existence of the two DPS components. In this calculation $\sigma_{e f f, 2 v 2}$ $=30 \mathrm{mb}$ and $\sigma_{e f f, 2 v 1}=15 \mathrm{mb}$.

single parton scattering one (dash-dotted line). The sum of the two contributions is represented by the solid line. We get a reasonable agreement with the LHCb experimental data [32.

In the presence of the (single) parton splitting $(2 \mathrm{v} 1)$ contribution the situation becoming more complicated [57]. It was shown that the ratio of the 2v1-to-2v2 depends on rapidity of produced quark/antiquark and collision energy. In Fig. 7 we show the empirical $\sigma_{e f f}$, for double charm production in the case when conventional and single parton splitting contributions are added together. The effective parameter $\sigma_{\text {eff }}$ rises with the centre-of-mass energy. A sizeable difference of results for different choices of scales can be observed in addition. Observation of such an effect would require very precise experimental data for a few center of mass energies.

Now we proceed to the jets with large rapidity separation. In Fig. 8 we show distribution in the rapidity distance between two jets in leading-order 
collinear calculation and between the most distant jets in rapidity in the case of four DPS jets. In this calculation we have included cuts for the CMS expriment [50]: $y_{1}, y_{2} \in(-4.7,4.7), p_{1 t}, p_{2 t} \in(35 \mathrm{GeV}, 60 \mathrm{GeV})$. For comparison we show also results for the BFKL calculation from Ref. [47. For this kinematics the DPS jets give sizeable (relative) contribution only at large rapidity distance. The NLL BFKL cross section (long-dashed line) is smaller than that for the LO collinear approach (short-dashed line).
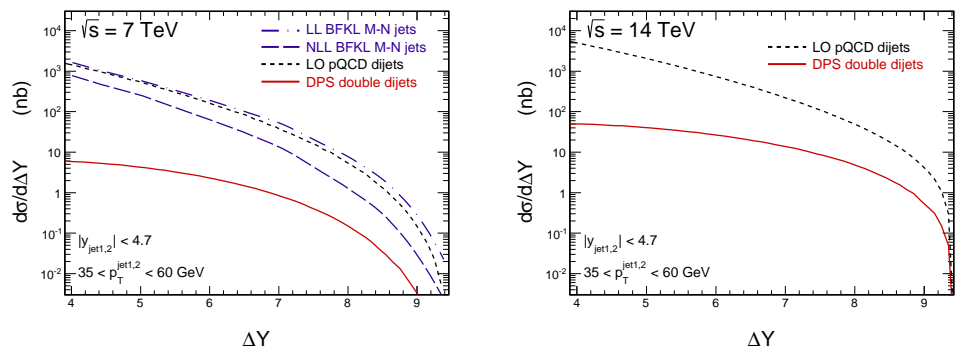

Fig. 8. Distribution in rapidity distance between jets $\left(35 \mathrm{GeV}<p_{t}<60 \mathrm{GeV}\right)$. The collinear pQCD result is shown by the short-dashed line and the DPS result by the solid line for $\sqrt{s}=7 \mathrm{TeV}$ (left panel) and $\sqrt{s}=14 \mathrm{TeV}$ (right panel). For comparison we show also results for the BFKL Mueller-Navelet jets in leadinglogarithm and next-to-leading-order logarithm approaches from Ref. [47.

In Fig. 9 we show rapidity-diffference distribution for even smaller lowest transverse momenta of the "jet". A measurement of such minijets may be, however, difficult. Now the DPS contribution may even exceed the standard SPS dijet contribution, especially at the nominal LHC energy. How to measure such (mini)jets is an open issue. In principle, one could measure correlations of semihard $\left(p_{t} \sim 10 \mathrm{GeV}\right)$ neutral pions with the help of socalled zero-degree calorimeters (ZDC).

Finally we wish to only briefly mention the situation for production of two gauge bosons, e.g. $W^{+} W^{-}$pairs. Many mechanisms contribute in this reaction [53]. How the situation may look at future high-energy experiments at the LHC and FCC is illustrated in Table 1. In this calculation we assumed $\sigma_{\text {eff }}=15 \mathrm{mb}$. Such an empirical value was extracted from phenomenological studies of high-energy processes (also at the LHC) that are, however, dominated rather by gluon-gluon processes. Whether a corresponding value of $\sigma_{\text {eff }}$ for processes dominated by quark-antiquark annihilation $\left(D_{q \bar{q}^{\prime}} \mathrm{dPDF}\right.$ in the cross section formula) is similar is not completely clear at present. For reference we show the leading-order contribution due to quark-antiquark annihilation. Clearly the relative contribution of DPS is growing with collision energy. The DPS contribution has slightly differ- 

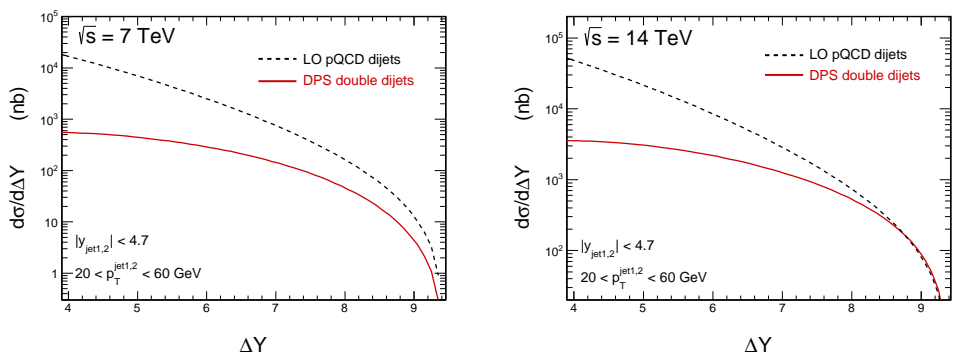

Fig. 9. The same as in the previous figure but now for smaller lower cut on minijet transverse momentum.

Table 1. Cross section for $W^{+} W^{-}$production at different collision energies for the dominant $q \bar{q}$ and DPS contributions.

\begin{tabular}{|l|c|c|}
\hline & $q \bar{q}$ & DPS \\
\hline 8000 & 0.032575 & $0.1775(-03)$ \\
14000 & 0.06402 & $0.6367(-03)$ \\
100000 & 0.53820 & 0.03832 \\
\hline
\end{tabular}

ent characteristics of the final state than other contributions [53]. Detailed studies require to go rather to leptons (electrons, muons), study differential distributions and consider background contributions. This issue was discussed in the literature only briefly (see e.g. [52, 66]).

\section{Conclusions}

We have shown that the cross section for $c \bar{c} c \bar{c}$ production grows much faster than the cross section for $c \bar{c}$ production making the production of two pairs of $c \bar{c}$ particularly attractive in the context of exploring the doubleparton scattering processes.

We have also shown predictions for production of $c \bar{c} c \bar{c}$ in the doubleparton scattering in the factorized Ansatz with each step calculated in the $k_{t}$-factorization approach. We have presented also cross sections for the production of $D^{0} D^{0}$ (or $\bar{D}^{0} \bar{D}^{0}$ ) pairs of mesons. The results of the calculation have been compared to recent results of the LHCb collaboration. The best agreement with the LHCb data was obtained for the Kimber-Martin-Ryskin UGDF. This approach, as discussed already in the literature, effectively includes higher-order QCD corrections. Rather good agreement was obtained for transverse momentum distribution of $D^{0}\left(\bar{D}^{0}\right)$ mesons and $D^{0} D^{0}$ invari- 
ant mass distribution. The distribution in azimuthal angle between both $D^{0}$ 's suggests that some contributions may be still missing or the rather crude approximation used in calculating DPS are not sufficient.

We have discussed also so-called parton splitting mechanism in the context of the $c \bar{c} c \bar{c}$ production. The sum of the $2 \mathrm{v} 1$ and $2 \mathrm{v} 2$ contributions behaves almost exactly like the $2 \mathrm{v} 2$ contribution, This leads e.g. to effective energy dependence of $\sigma_{\text {eff }}$.

We have discussed also how the double-parton scattering effects may contribute to large-rapidity-distance dijet correlations. Already leading-order calculation provides quite adequate description of inclusive jet production when confronted with recent results obtained by the ATLAS and CMS collaborations [51]. We have shown distributions in rapidity distance between the most-distant jets in rapidity. The relative contribution of the DPS mechanism increases with increasing distance in rapidity between jets. We have also shown some recent predictions of the Mueller-Navelet jets in the LL and NLL BFKL framework. For the CMS configuration our DPS contribution is smaller than the dijet SPS contribution and only slightly smaller than that for the NLL BFKL calculation. We have demonstrated that the relative effect of DPS can be increased by lowering the transverse momenta. A four-jet final states analyses of distributions in rapidity distance and other kinematical observables was performed by us very recently [65.

Finally we have also mentioned the role of DPS effects in inclusive production of $W^{+} W^{-}$pairs. We have shown that the relative contribution of DPS grows with collision energy. In experiments one measures electrons or muons. Whether experimental identification of the DPS contribution in this case is possible requires detailed Monte Carlo studies.

\section{REFERENCES}

[1] P.V. Landshoff and J.C. Polinghorne, Phys. Rev. D18 (1978) 3344.

[2] F. Takagi, Phys. Rev. Lett. 18 (1979) 1296.

[3] C. Goebel and D.M. Scott and F. Halzen, Phys. Rev. D22 (1980) 2789.

[4] B. Humpert, Phys. Lett. B131 (1983) 461.

[5] N. Paver and D. Treleani, Phys. Lett. B146 (1984) 252.

[6] N. Paver and D. Treleani, Z. Phys. C28 (1985) 187.

[7] M. Mekhfi, Phys. Rev. D32 2371; M. Mekhfi, Phys. Rev. D32 2380.

[8] B. Humpert and R. Oderico, Phys. Lett. B154 (1985) 211.

[9] T. Sjöstrand and M. van Zijl, Phys. Rev. D36 (1987) 2019.

[10] M. Drees and T. Han, Phys. Rev. Lett. 77 (1996) 4142.

[11] A. Kulesza and W.J. Stirling, Phys. Lett. B475 (2000) 168. 
[12] A. Del Fabbro and D. Treleani, Phys. Rev. D66 (2002) 074012.

[13] E.L. Berger, C.B. Jackson and G. Shaughnessy, Phys. Rev. D81 014014 (2010).

[14] J.R. Gaunt, C-H. Kom, A. Kulesza and W.J. Stirling, arXiv:1003.3953.

[15] M. Strikman and W. Vogelsang, Phys. Rev. D83 (2011) 034029.

[16] B. Blok, Yu. Dokshitzer, L. Frankfurt and M. Strikman, Phys. Rev. D83 (2011) 071501.

[17] C.H. Khom, A. Kulesza and W.J. Stirling, Phys. Rev. Lett. 107 (2011) 082002 .

[18] S.R. Baranov, A. M. Snigirev and N.P. Zotov, arXiv:1105.6279.

[19] A.M. Snigirev, Phys. Rev. D68 (2003) 114012.

[20] V.L. Korotkikh and A.M. Snigirev, Phys. Lett. B594 (2004) 171.

[21] T. Sjöstrand and P.Z. Skands, JHEP 0403 (2004) 053.

[22] J.R. Gaunt and W.J. Stirling, JHEP 1003 (2010) 005.

[23] J.R. Gaunt and W.J. Stirling, JHEP 1106 (2011) 048.

[24] M. Diehl and A. Schäfer, Phys. Lett. B698 (2011) 389.

[25] M.G. Ryskin and A.M. Snigirev, Phys. Rev. D83 (2011) 114047.

[26] M. Diehl, D. Ostermeier and A. Schäfer, JHEP 1203 (2012) 089.

[27] A.V. Manohar and W.J. Waalewijn, Phys. Rev. D85 (2012) 114009.

[28] A.V. Manohar and W.J. Waalewijn, Phys. Lett. B713 (2012) 196.

[29] M. Diehl and T. Kasemets, Phys.Rev. D91 (2015) 014015.

[30] M. Diehl, T. Kasemets and S. Kane, JHEP1405 (2014) 118.

[31] M. Łuszczak, R. Maciuła and A. Szczurek, Phys. Rev. D85 (2012) 094034.

[32] R. Aaij et al. [LHCb Collaboration], J. High Energy Phys. 06, 141 (2012); J. High Energy Phys 03, 108 (2014); arXiv:1205.0975 [hep-ex]].

[33] R. Maciuła and A. Szczurek, Phys. Rev. D87 (2013) 074039.

[34] A. H. Mueller, and H. Navelet, Nucl. Phys. B282, 727 (1987).

[35] V. Del Duca and C. R. Schmidt, Phys. Rev. D49, 4510 (1994); arXiv:9311290 [hep-ph].

[36] W. J. Stirling, Nucl. Phys. B423, 56 (1994); arXiv:9401266 [hep-ph].

[37] V. Del Duca and C. R. Schmidt, Phys. Rev. D51, 2150 (1995); arXiv:9407359 [hep-ph].

[38] V. T. Kim and G. B. Pivovarov, Phys. Rev. D 53, 6 (1996); arXiv:9506381 [hep-ph].

[39] J. Andersen, V. Del Duca, S. Frixione, C. Schmidt and W. J Stirling, J. High Energy Phys. 02, 007 (2001); arXiv:0101180 [hep-ph].

[40] J. Bartels, D. Colferai and G. Vacca, Eur. Phys. J. C24, 83 (2002); Eur. Phys. J. C 29, 235 (2003).

[41] A. Sabio Vera and F. Schwennsen, Nucl. Phys. B776, 170 (2007); arXiv:0702158 [hep-ph]. 
[42] C. Marquet and C. Royon, Phys. Rev. D79, 034028 (2009); arXiv:0704.3409 [hep-ph].

[43] D. Colferai, F. Schwennsen, L. Szymanowski and S. Wallon, J. High Energy Phys. 12, 026 (2010); arXiv:1002.1365 [hep-ph].

[44] F. Caporale, D. Y. Ivanov, B. Murdaca, A. Papa and A. Perri, J. High Energy Phys. 02, 101 (2012); arXiv:1112.3752 [hep-ph].

[45] D. Y. Ivanov and A. Papa, J. High Energy Phys. 05, 086 (2012); arXiv:1202.1082 [hep-ph].

[46] F. Caporale, D. Y. Ivanov, B. Murdaca and A. Papa, Nucl. Phys. B877, 73 (2013); arXiv:1211.7225 [hep-ph].

[47] B. Ducloue, L. Szymanowski and S. Wallon, J. High Energy Phys. 05, 096 (2013); arXiv:1302.7012 [hep-ph].

[48] B. Duclou, L. Szymanowski and S. Wallon, Phys. Rev. Lett. 112, 082003 (2014); arXiv:1309.3229 [hep-ph].

[49] V. Del Duca, L. J. Dixon, C. Duhr, J. Pennington, J. High Energy Phys. 02, 086 (2014): arXiv:1309.6647.

[50] I. Pozdnyakov, private communication

[51] R. Maciula and A. Szczurek, Phys. Rev. D90 (2014) 014022.

[52] J.R. Gaunt, Ch.-H. Kom, A. Kulesza and W.J. Stirling, Eur. Phys. J. C69 (2010) 53, arXiv:1003.3953 [hep-ph].

[53] M. Luszczak, A. Szczurek and C. Royon, J. High Energy Phys. 02, 098 (2015); arXiv:1409.1803 [hep-ph]].

[54] K. Golec-Biernat and E. Lewandowska, Phys. Rev. D90 (2014) 094032.

[55] W. Krasny and W. Płaczek, Acta Phys. Pol. B45 (2014) 71, arXiv:1305.1769 [hep-ph].

[56] G. Calucci and D. Treleani, Phys. Rev. D60 (1999) 054023.

[57] J.R. Gaunt, R. Maciula and A. Szczurek, Phys. Rev. D90 (2014) 054017.

[58] R. Maciula and A. Szczurek, Phys. Rev. D 90, 014022 (2014) [arXiv:1403.2595 [hep-ph]].

[59] R. Aaij et al. [LHCb Collaboration], Phys. Lett. B 707, 52 (2012) arXiv:1109.0963 [hep-ex]].

[60] G. Aad et al. [ATLAS Collaboration], New J. Phys. 15, 033038 (2013) arXiv:1301.6872 [hep-ex]].

[61] W. Schäfer and A. Szczurek, Phys. Rev. D85 (2012) 094029.

[62] A. van Hameren, R. Maciula and A. Szczurek, Phys. Rev. D 89, 094019 (2014); arXiv:1402.6972 [hep-ph].

[63] A. Donnachie and P.V. Landshoff, Phys. Lett. B296 (1992) 227.

[64] A. van Hameren, R. Maciuła and A. Szczurek, arXiv:1504.06490 [hep-ph].

[65] R. Maciula and A. Szczurek, arXiv:1503.08022 [hep-ph].

[66] arXiv:1504.06491 [hep-ph], a talk at the EPIPHANY2015 conference, January 2015, Kraków, Poland. 